Document downloaded from:

http://hdl.handle.net/10251/95467

This paper must be cited as:

Santonja Blasco, L.; Moriana Torró, R.; Badia, J.; Ribes-Greus, A. (2010). Thermal analysis applied to the characterization of degradation in soil of polylactide: I. Calorimetric and viscoelastic analyses. Polymer Degradation and Stability. 95(11):2185-2191.

doi:10.1016/j.polymdegradstab.2010.08.005

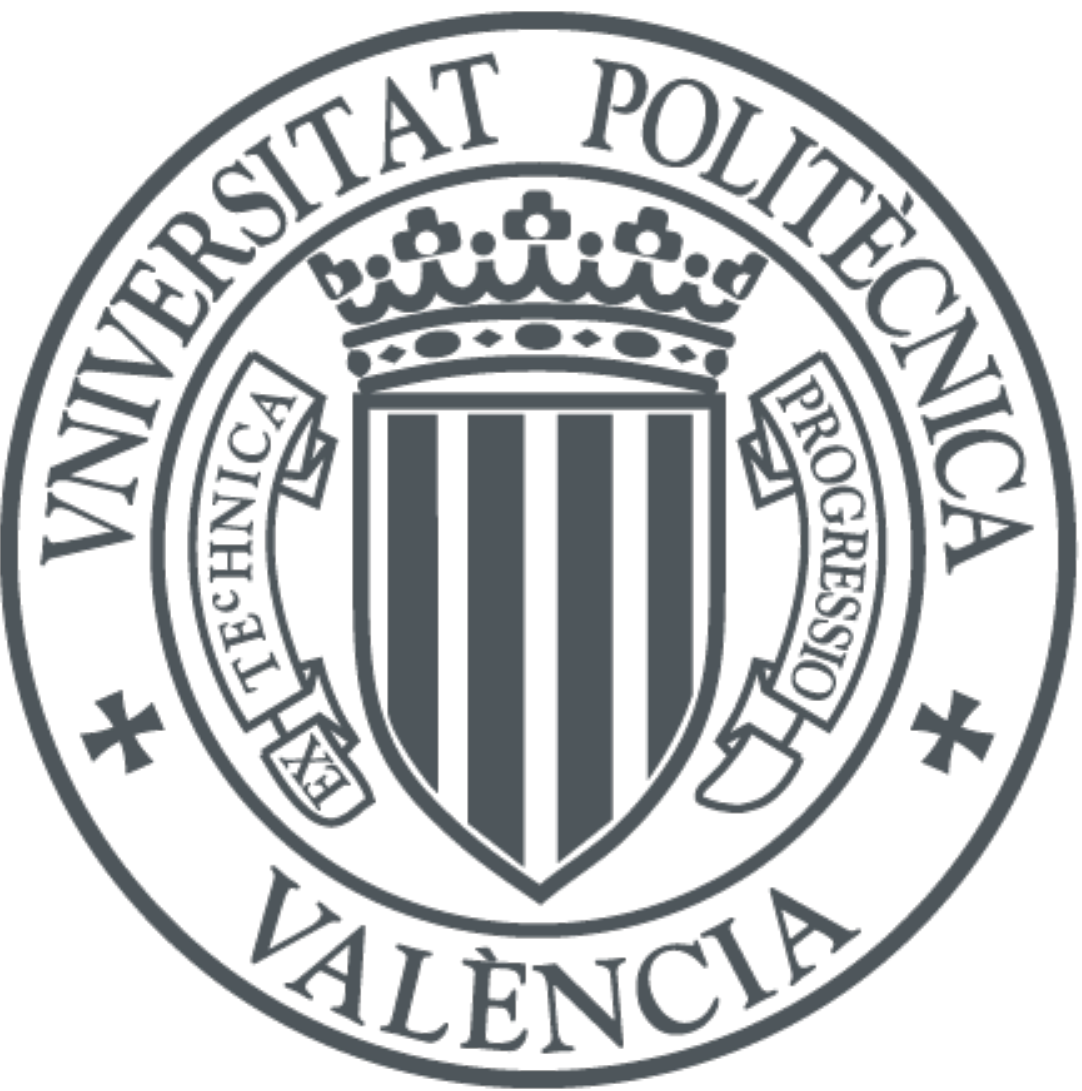

The final publication is available at

http://doi.org/10.1016/j.polymdegradstab.2010.08.005

Copyright Elsevier

Additional Information 
THERMAL ANALYSIS APPLIED TO THE CHARACTERIZATION OF

DEGRADATION IN SOIL OF POLYLACTIDE: I. CALORIMETRIC AND VISCOELASTIC ANALYSES

L. Santonja-Blasco, Rosana Moriana, J.D. Badía, and A. Ribes-Greus*

This is an open-access version, according to http://www.sherpa.ac.uk/romeo/issn/0141-3910/

Full text available at https://www.sciencedirect.com/science/article/pii/S0141391010003381

DOI: https://doi.org/10.1016/j.polymdegradstab.2010.08.005

Please, cite it as:

L. Santonja-Blasco, Rosana Moriana, J.D. Badía, A. Ribes-Greus. Thermal analysis applied to the characterization of degradation in soil of polylactide: I. Calorimetric and viscoelastic analyses. Polymer Degradation and Stability 2010, 95(11):2185-2191

Instituto de Investigación en Tecnología de Materiales.

Universidad Politécnica de Valencia,

Camino de Vera s/n 46071 Valencia, Spain

*To whom correspondence should be addressed.

Corresponding author. Fax: +34963879817

E-mail address: aribes@ter.upv.es 


\title{
THERMAL ANALYSIS APPLIED TO THE CHARACTERIZATION OF \\ DEGRADATION IN SOIL OF POLYLACTIDE: I. CALORIMETRIC AND \\ VISCOELASTIC ANALYSES
}

\author{
L. Santonja-Blasco, Rosana Moriana, J.D. Badía, and A. Ribes-Greus*
}

\begin{abstract}
An accelerated soil burial test has been performed on a commercial polylactide (PLA) for simulating non-controlled disposal. Degradation in soil promotes physical and chemical changes in polylactide properties, which can be characterized by Thermal Analysis techniques. Physical changes occurred in polylactide due to the degradation in soil were evaluated by correlating their calorimetric and viscoelastic properties. It is highly remarkable that each calorimetric scan offer specific and enlightening information. Degradation in soil affects the polylactide chains reorganization. A multimodal melting behaviour is observed for buried PLA, since degradation in soil also promotes the segregation of the crystalline phase, enlarging the lamellar thickness distribution of the population with bigger average size. Morphological changes lead to an increase in the free volume of the polylactide chains in the amorphous phase that highly affected the bulk properties. Thermal Analysis techniques provide reliable indicators of the degradation stage of polylactide induced by degradation in soil, as corroborated by molecular weight analysis.
\end{abstract}

Keywords: polylactide (PLA), degradation in soil, differential scanning calorimetry (DSC), dynamical-mechanical-thermal analysis (DMTA), free-volume, lamellar thickness distribution. 


\section{Introduction}

Polylactide (PLA) is an aliphatic, biodegradable, and compostable polyester which can be easily processed with standard equipment to yield articles that can be used in many applications such as in industrial packaging, in the building area, in medical, agriculture and textile field, etc [1,2,3]. Initially, polylactide products were produced for biomedical purposes and thus their hydrolysis processes captured the whole research attention [4-5]. Studies performed in neutral media such as phosphate-buffered solution, in vivo solution and water, have been extensively analyzed in order to determine the hydrolytic degradation mechanism $[6,7,8,9,10]$. Nowadays PLA stands out as a reliable alternative to commodities in packaging applications. This solution will therefore imply an increase of a new source of plastic waste. Hence, to correctly manage the PLA disposal, its biodegradability performance has been studied in several environmental conditions, such as composting, microbiological cultures, biological degradation and disposal in soil $[11,12,13,14]$.

Extensive work has been performed by several researchers for understanding the degradation in soil of polymers. Former PLA biodegradation studies stated that hydrolytic reactions seem to act in the initial stage of the overall PLA biodegradation, proceeding by chain-end scission in the PLA matrix, which eases the successive biotic assimilation $[11,15,16,17]$. A biotic environment implies chain scissions and the physical and chemical properties of the polymer can be severely modified. Thus, the common characterization is mainly carried out by means of the measurement of the molecular weight or the weight loss changes. Ho et al. found that about $20 \%$ of a PLA film was mineralised to $\mathrm{CO}_{2}$ after 182 days in a laboratory respirometer charged with soil at $28^{\circ} \mathrm{C}$ [18]. Calmon et al. found that PLA films had weight losses varying from 0 to $100 \%$ after burial in soil for 24 months depending on PLA type and location [19]; in 
contrast Urayama et al. only found a decrease of a $20 \%$ in molecular weight of PLA (100\% L) plates after 20 months in soil [14]. In addition, it has been suggested that traditional techniques as the measurement of the weight loss changes for studying polymer biodegradation have some limitations especially after 3 months, because of the adhesion of soil and fungi to the polymer, which can mask real results and thus induce misleading information [14,20,21]. Fast, cost-effective and reliable characterization procedures for testing the biodegradation effects on polymers should be developed and implemented. Thermal Analysis techniques have been successfully applied in our research group to monitor and control the degradation effects on the macroscopic properties of polymers submitted to different degradative environments since they offer a huge amount of parameters that can act as indicators of the extent of degradation [22,23,24,25,26]. Figure 1 summarizes the Thermal Analysis techniques proposed for the study of the extent of degradation on PLA: Thermogravimetry (TGA), Differential Scanning Calorimetry (DSC) and Dynamic-Mechanical-Thermal Analysis (DMTA), as well as the principal parameters selected for the study. The first paper is focused on the DSC and DMTA study and the second one on the TGA.

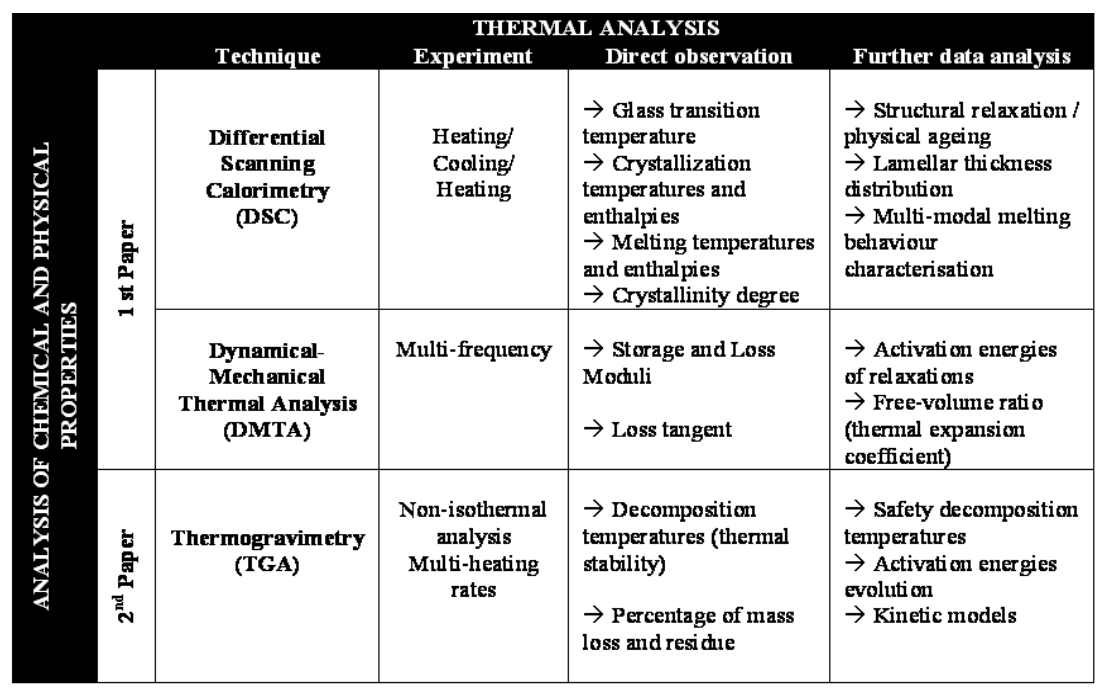

Figure 1: Summary of parameters used to assess the extent of degradation in soil on polylactide. 
The aim of this work is to simulate the degradation in the environmental conditions that PLA is subjected during non-controlled disposal. In this set of papers, physical changes occurred to polylactide properties throughout the degradation in soil process are analysed by Thermal Analysis, making efforts on establishing new insights in studying the degradation in soil process on polymers. The study is complemented with the analysis of the evolution of the average molecular weight in number and weight by Gel Permeation Chromatography, aiming to test the reliability and consistency of the techniques proposed in the assessment of degradation in soil effects on PLA.

\section{Experimental section}

\subsection{Material and sample preparation}

A commercial polylactide (PLA), obtained from renewable resources by ring opening polymerization supplied by Natureworks (Minnetonka, USA) was used in this study. This PLA is a commercial resin with $3.8 \%$ meso-lactide and with a numberaverage molecular weight of $102.230 \mathrm{~g} / \mathrm{mol}$, as measured by Gel Permeation Chromatography.

PLA pellets were previously dried with demoisturized air at $80^{\circ} \mathrm{C}$ during 4 hours. Rectangular plates were prepared by melt compression in a Collin PCS-GA Type Press 800 (GA, USA) at an initial temperature of the hot plates of $195^{\circ} \mathrm{C}$ and final temperature of $60^{\circ} \mathrm{C}$. Five pressure steps were performed as follows starting with an 5 minutes at 6 bar, 8 minutes at 75 bar, 8 minutes at 155 bar, 4 minutes at 215 bar, and 11 minutes at 45 bar. Specimens of $145 \times 10 \times 2 \mathrm{~mm}$ were cut from the melt-pressed plates for the degradation in soil tests. Since this work approaches the degradation in soil of non-controlled landfilling of consumer goods, which are obtained by means of, at least, one processing step, “non-buried PLA” has been considered the reference material of the study. 


\subsection{Accelerated soil burial test}

PLA plates were subjected to a controlled degradation in soil test under controlled conditions (temperature, water content and pH), following the ISO 846-1997 International Norm, method D [27]. Samples were buried in biologically active soil and kept in a Heraeus B12 (Hanau, Germany) culture oven at $28{ }^{\circ} \mathrm{C}$. The soil used in these tests was a red soil extract taken from a culture field in Alginet (Valencia). Microbial activity of soil was monitored with cotton along the extension of the experiment. The soil was maintained at approximately $\mathrm{pH} 7$ and a relative humidity of $0.87 \mathrm{~g}$ water/g wet soil. To ensure the oxygenation of the soil, a protocol of periodical air oxygen supply was followed. Test specimens were extracted at 30, 150, 300 and 450 days, cleaned and kept in a desiccator during 4 days in order to ensure water desorption before being analyzed.

\subsection{Analytic procedures}

Samples were thermally characterized by means of Differential Scanning Calorimetry (Mettler Toledo DSC822, Columbus, OH, USA). The calibration of the DSC was previously checked by In and Zn standards. Three calorimetric scans were performed to each sample at a heating/cooling rate of $10^{\circ} \mathrm{C} / \mathrm{min}$. Samples of around 4 mg were introduced in a pierced aluminium crucible, with capacity for $40 \mu \mathrm{L}$. The first heating scan, in which the thermal history influence is presented, was performed from 0 to $200^{\circ} \mathrm{C}$; the cooling scan went from $200^{\circ} \mathrm{C}$ to $0^{\circ}$ and the second heating scan from 0 to $200^{\circ} \mathrm{C}$. All experiments were performed under $\mathrm{N}_{2}$ dry gas as protective gas ( $50 \mathrm{ml} / \mathrm{min}$ ) to avoid the water condensation in the equipment and purged with $\mathrm{N}_{2}(200 \mathrm{ml} / \mathrm{min})$ in the furnace.

The mechanical and viscoelastic properties were assessed by means of a Rheometric Scientifics Dynamic-Mechanical-Thermal Analyser Mark IV (USA). The 
deformation force was set at $0.01 \mathrm{~N}$. The displacement was checked before all the experiments. Experiments were performed by using dual cantilever clamping in bending mode. Specimens of $40 \times 10 \times 2 \mathrm{~mm}$ were heated from 35 to $150^{\circ} \mathrm{C}$ in iso-step mode every $2^{\circ} \mathrm{C}$ in the frequency ( $f$ ) range from 0.1 to $39 \mathrm{~Hz}$ measuring 5 points per decade. In order to correlate the results obtained by Thermal Analysis with the molecular weight changes, the number and weight average molecular weights ( $\bar{M}_{n}$ and $\bar{M}_{w}$ respectively) of the samples were evaluated in tetrahydrofuran (THF) at $25^{\circ} \mathrm{C}$ by a GPC Agilent 1100 Series, using a PL Gel $5 \mu \mathrm{m} 104 \AA$ column, of 300 x 7.5 mm, from Polymer Laboratories.

\section{Results and discussion}

The extent of biodegradation of polylactide (PLA) has been deeply characterized by means of Differential Scanning Calorimetry (DSC) and Dynamical-MechanicalThermal Analysis (DMTA) experiments. A parallel DSC/DMTA results interpretation along the study will thus provide specific indicators to monitor the extent of degradation, by understanding the role of both amorphous and crystalline fractions of PLA. Furthermore, these results have been associated with the average molecular weight evolution in order to validate the suitability of Thermal Analysis techniques for monitoring degradation in soil on PLA.

Figures $2 \mathrm{a}$ and $2 \mathrm{~b}$ show the calorimetric thermogram and the mechanical relaxation spectrum of non-buried PLA, respectively. In the Figure 2a, the three calorimetric (heating, cooling, and second heating) scans are plotted. From the first heating scan, the degradation in soil effect on PLA was assessed, since it represents the current status of the buried polymer. The following transitions are observed along the increasing temperature-axis: glass transition (between $40^{\circ} \mathrm{C}-75^{\circ} \mathrm{C}$ ), cold crystallization (between $90^{\circ} \mathrm{C}-140^{\circ} \mathrm{C}$ ) and melting process (between $140^{\circ} \mathrm{C}-160^{\circ} \mathrm{C}$ ). From each 
calorimetric transition, sensitive indicators were studied to evaluate degradation. The cooling DSC scan only shows the glass transition and the second DSC heating scan shows the glass transition, overlapped with the structural relaxation enthalpy. In the second heating scan, PLA has different thermal history than the shown at the first scan; because the amorphous phase does not crystallize after cooling the material at $10^{\circ} \mathrm{C} / \mathrm{min}$.
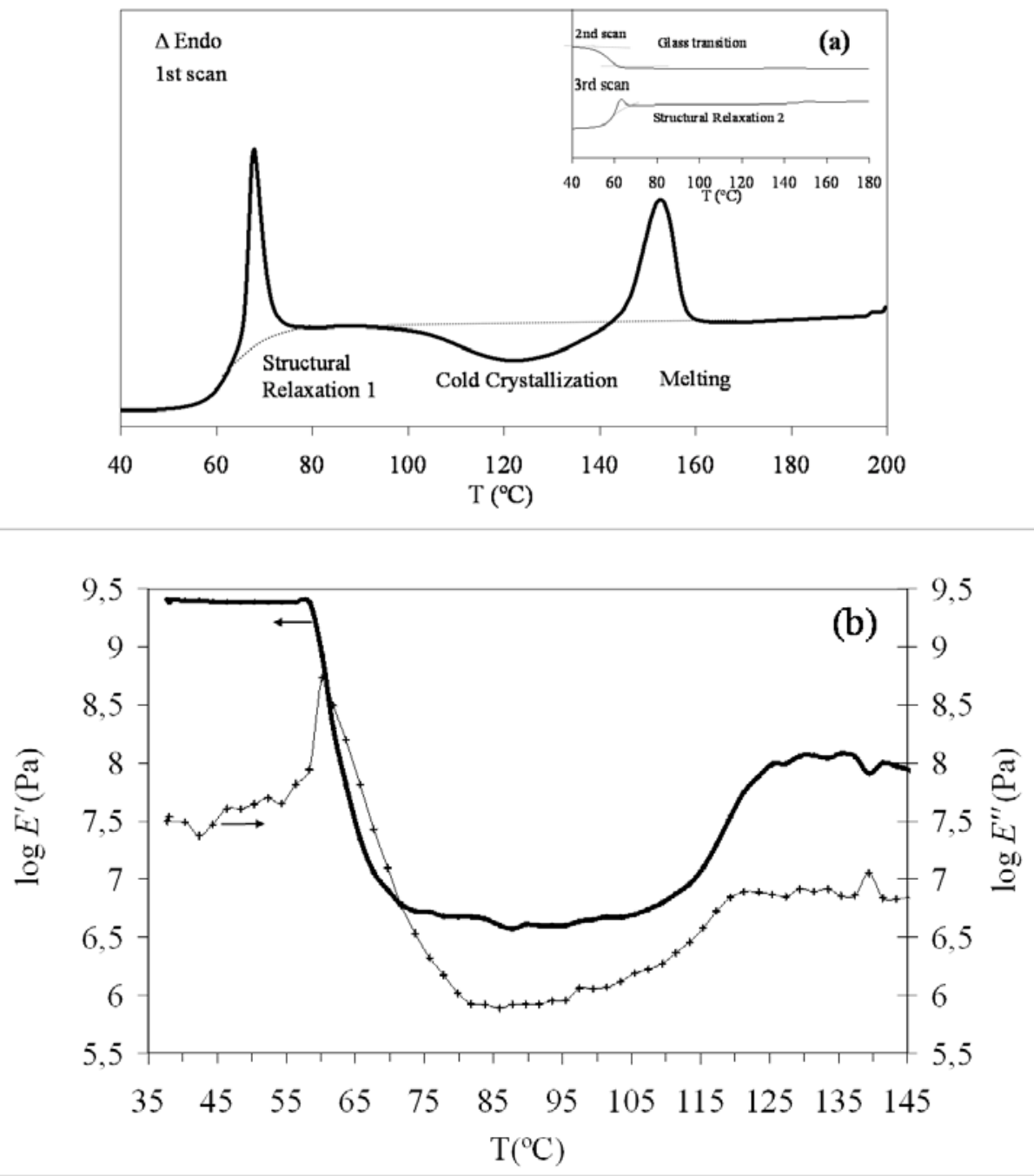

Figure 2: Calorimetric and viscoelastic behavior of non-buried polylactide (a)

calorimetric thermogram and (b) $\log \left(E^{\prime}\right)$ and $\log \left(E^{\prime \prime}\right)$ versus temperature.

In the Figure $2 \mathrm{~b}$ the storage $\left(E^{\prime}\right)$ and loss ( $\left.E^{\prime \prime}\right)$ moduli versus temperature at the commonly used frequency of $1 \mathrm{~Hz}$ are plotted. Similar curves are obtained for all the other frequencies between 0.1 and $39 \mathrm{~Hz}$, but are not displayed for the sake of clarity. 
The mechanical relaxation spectra show different relaxation zones which can be assigned to the calorimetric transitions along the increasing temperature-axis: glass/rubber transition $\left(55-75^{\circ} \mathrm{C}\right)$, rubbery plateau $\left(75-90^{\circ} \mathrm{C}\right)$, rubber/crystallization transition $\left(90-140^{\circ} \mathrm{C}\right)$, and melting/flowing (from $140^{\circ} \mathrm{C}$ on).

Figure 3 shows the effect of degradation in soil on the first DSC heating scan. The degradation in soil modifies the storage and loss modulus spectra as can be seen at Figures $4 \mathrm{a}$ and $4 \mathrm{~b}$, respectively. Taking into account that the first calorimetric thermogram (first DSC scan) and the mechanical relaxation spectrum (DMTA scan) are directly related; the discussion of the results is focused in the three important transitions observed in the calorimetric thermogram and the corresponding mechanical relaxation spectrum.

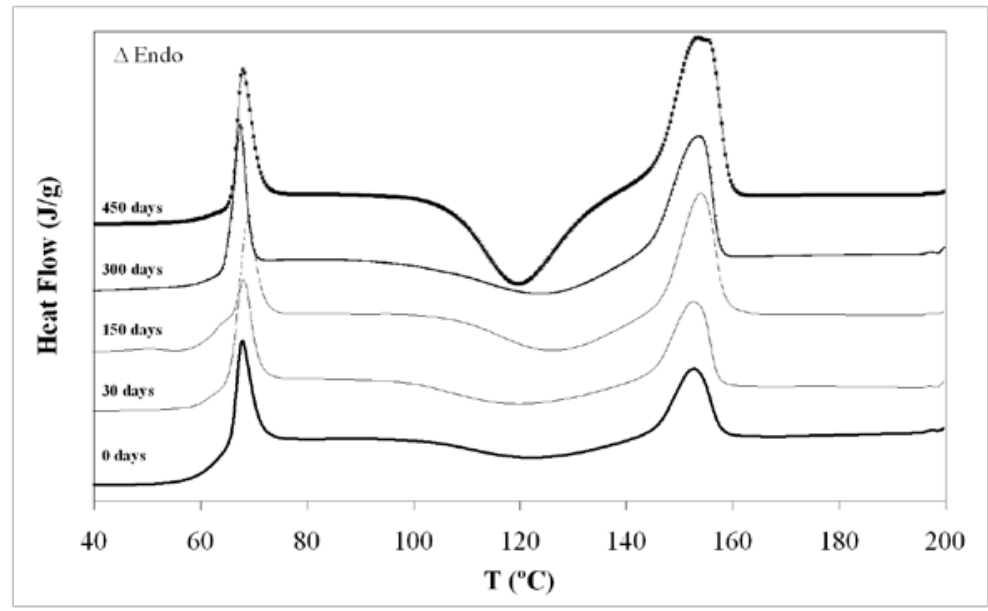

Figure 3: Comparative first scan of the calorimetric thermograms at different degradation times. 

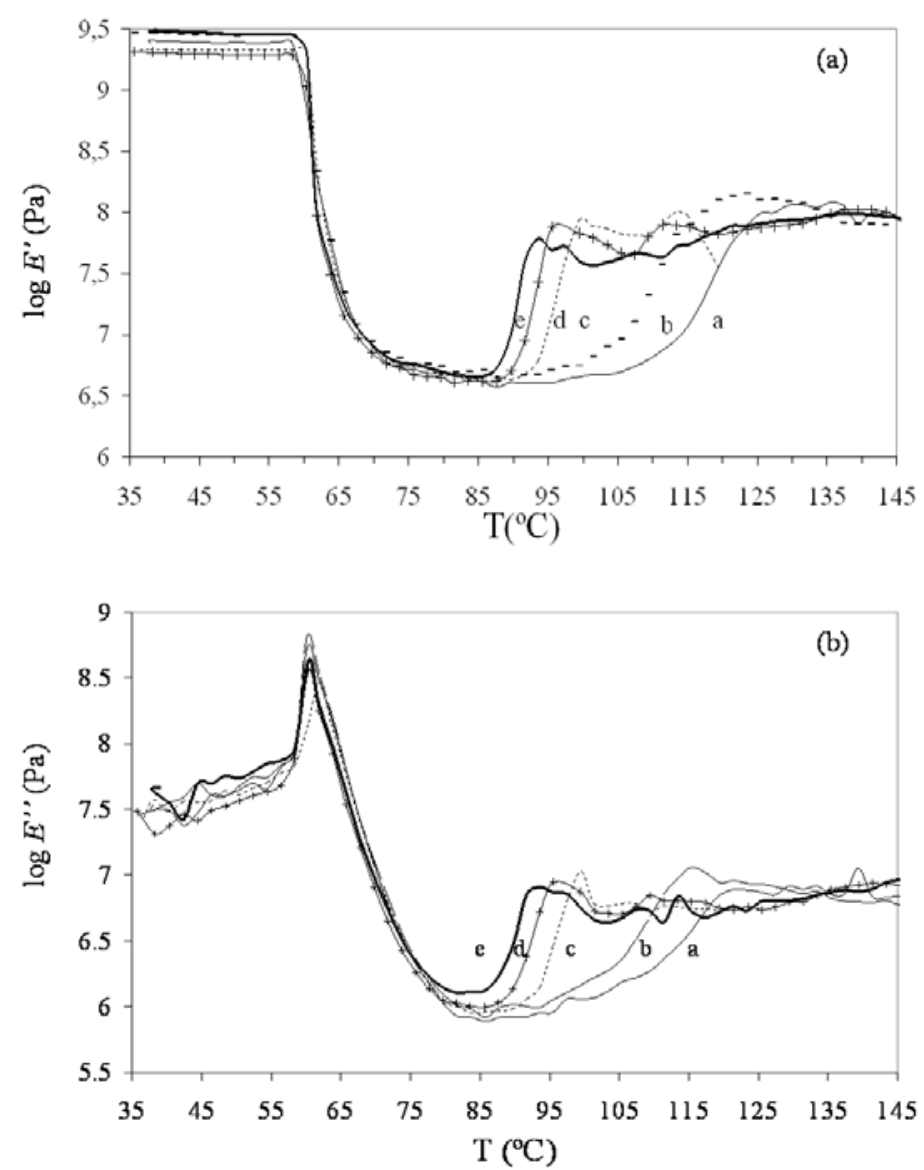

Figure 4: (a) Effect of degradation in soil on $\log E^{\prime}$ vs. temperature. (b) Effect of degradation in soil of polylactide on $\log E$ ', vs. temperature.( a. 0 days; b. 30 days; c. 150 days; $\mathrm{d} 300$ days; e. 450 days).

\subsection{Glass transition assessment}

The study of the glass transition region from the calorimetric and the mechanical techniques enables analyzing the effect of degradation on the amorphous molecular chains. At the first calorimetric scan (Figure 2) an endothermic phenomenon, the structural relaxation, overlapped to the glass transition relaxation, is observed at $61^{\circ} \mathrm{C}$. In order to assess this phenomenon, the relative structural relaxation enthalpy $\left(\Delta H_{R}\right)$ is proposed as indicator. The enthalpies were calculated by the specific area $(\mathrm{J} / \mathrm{g})$ of the consequent endotherm using a spline baseline. The relative structural relaxation enthalpy $\left(\Delta H_{R}\right)$ is obtained by the subtraction of the structural relaxation enthalpy of 
the first scan $\left(\Delta H_{R 1}\right)$ to the one related obtained in the second heating scan $\left(\Delta H_{R 3}\right)$. It is provided in relative terms with regards to the non-buried sample (sample“o”) to study the " $i$ " times of degradation in soil $\Delta H_{R i}=\left(\Delta H_{R 1 i}-\Delta H_{R 3 i}\right) /\left(\Delta H_{R 10}-\Delta H_{R 30}\right)$. Table 1 shows the changes of $\Delta H_{R}$. It can be seen that when the soil burial test advances, there is an increasing tendency of this indicator reaching a 70\% increase at 450 days.

The glass transition temperature $\left(T_{g}\right)$ is obtained from the second heating DSC scan in which this is the only phenomenon shown, due to the applied cooling rate does not allow crystallization. The $T_{g}$ is calculated as the temperature at the inflection point of the phenomenon, and for non-buried PLA it is located around $56^{\circ} \mathrm{C}$.

Table 1: Calorimetric and viscoelastic parameters relaxation related to the glass transition.

\begin{tabular}{cccc|c}
\hline \multicolumn{3}{c|}{ DSC } & DMTA \\
\hline $\begin{array}{c}\text { Time in } \\
\text { soil (days) }\end{array}$ & $T_{g}\left({ }^{\circ} \mathbf{C}\right)$ & $\begin{array}{l}\Delta H_{R 1}(\mathbf{J} / \mathbf{g})- \\
\Delta H_{R 3}(\mathbf{J} / \mathbf{g})\end{array}$ & $\Delta H_{R}(\mathbf{J} / \mathbf{g})$ & $T_{\max }\left({ }^{\circ} \mathbf{C}\right)$ \\
\hline $\mathbf{0}$ & $56.5 \pm 1.0$ & $4.3 \pm 0.1$ & $1.0+0.02$ & $60.9 \pm 0.5$ \\
$\mathbf{3 0}$ & $56.1 \pm 0.8$ & $4.7 \pm 0.1$ & $1.1+0.02$ & $60.9 \pm 0.3$ \\
$\mathbf{1 5 0}$ & $56.7 \pm 1.0$ & $5.2 \pm 0.2$ & $1.2+0.04$ & $62.0 \pm 0.7$ \\
$\mathbf{3 0 0}$ & $55.7 \pm 0.7$ & $6.0 \pm 0.3$ & $1.4+0.07$ & $60.7 \pm 0.2$ \\
$\mathbf{4 5 0}$ & $55.9 \pm 0.6$ & $7.3 \pm 0.3$ & $1.7+0.04$ & $60.8 \pm 0.7$ \\
\hline
\end{tabular}

The glass-rubber relaxation of PLA appears in DMTA as a drop of the storage modulus $\left(E^{\prime}\right)$ to very low values. The peak temperature $\left(T_{\max }\right)$ taken from the maximum of the loss modulus ( $E$ ') related to the glass transition gives a value of approx. $60{ }^{\circ} \mathrm{C}$ at the frequency of $1 \mathrm{~Hz}$. As was expected, the temperature related to the glass-rubber relaxation is higher than the calorimetric glass transition temperature. The values of $T_{g}$ and $T_{\max }$ obtained for PLA submitted to degradation in soil are shown at Table 1 . These 
parameters do not offer significant changes with burial, because are sensitive to largescale morphological changes. With the aim of assessing the morphological rearrangements of PLA amorphous chains, a closer inspection has been carried out.

The calculation of the Arrhenius maps has thus been performed (Figure 5) in order to predict the influence of the degradation in soil on the viscoelastic performance of PLA. As expected, the relationship of $\ln (f)$ and $T_{\max }^{-1}$ can be fitted to the VogelFulcher-Tamman-Hesse (VFTH) equation [28]:

$$
\ln f=A-m_{v} \cdot \frac{1}{T_{\max }-T_{\infty}}
$$

where $f$ represent the selected frequencies in $\mathrm{Hz}, T_{\max }$ in $\mathrm{K}$, is the temperature obtained at the maximum value of $E$ ', $m_{v}=B / \alpha_{f}$ the slope of the equation, being $\alpha_{f}$ the thermal expansion coefficient and $B \cong 1, T_{\infty}$ is the temperature at which the free volume would be zero and $A$ is a pre-exponential factor. The thermal expansion coefficient trend during the degradation in soil for all samples is shown in Figure 6. The thermal expansion coefficient presents an increasing tendency when degradation time becomes longer. The increase is more noticeable from 300 days on.

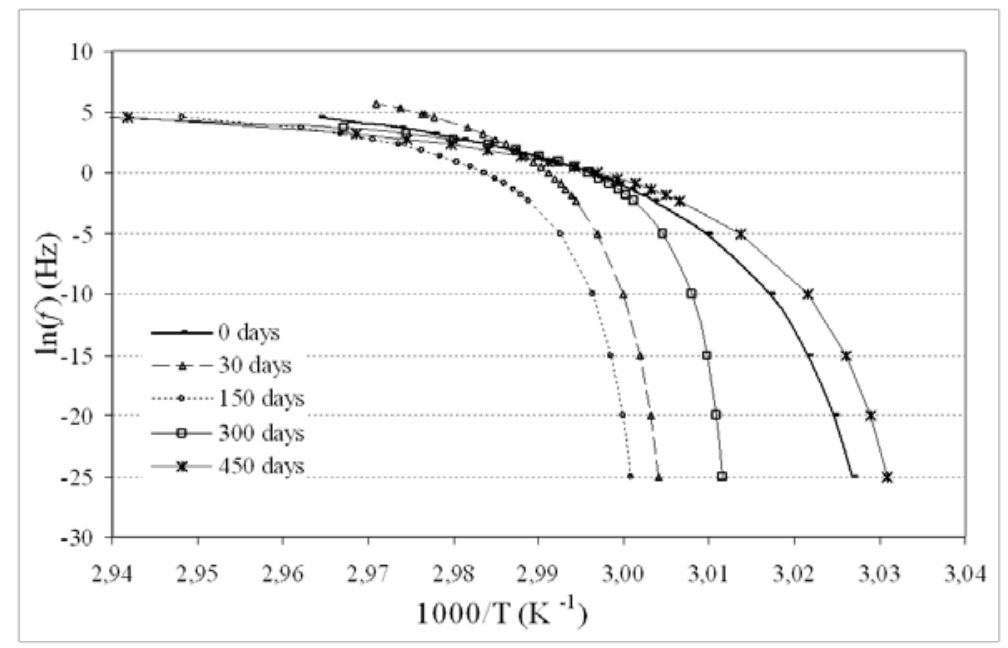

Figure 5: Arrhenius Maps obtained from multi-frequency DMTA analysis 


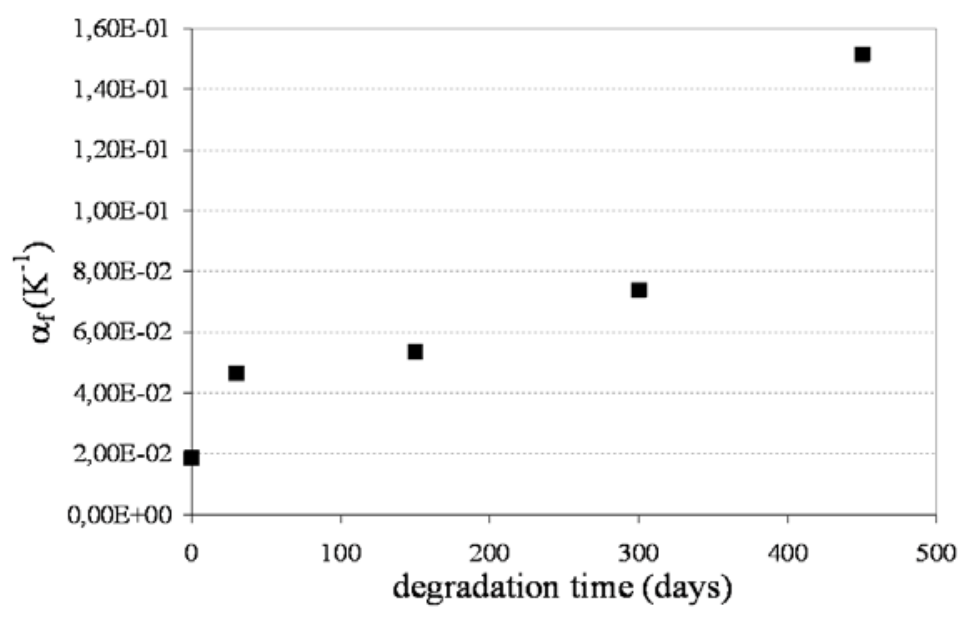

Figure 6: Thermal expansion coefficient vs. degradation time

Therefore, the free volume existing among the amorphous chains is higher as the extent of degradation rises up. This may be a direct consequence of chain cleavages in the amorphous phase, caused by degradation in soil agents (water and microorganisms) on the PLA matrix. Specifically in the presence of water it has been found to proceed through two alternative mechanisms: surface or heterogeneous, and bulky or homogeneous erosion [29].The free volume of the studied PLA arises with degradation in soil strengthening the suggestion of the higher compression as degradation in soil advances.

\subsection{Cold-crystallization evaluation}

When the glass transition relaxation is overcome other phenomenon very significant presented by PLA is the cold crystallization. In the first heating DSC scan, the cold crystallization is observed in an onset $\left(T_{0 n}\right)$ around $85^{\circ} \mathrm{C}$, since the chains which were constrained have the condition to freely crystallize and at around $123^{\circ} \mathrm{C}$ the exothermic peak $\left(T_{c}\right)$ is situated. Mechanical relaxation spectrum shows that the storage modulus increases again after the glass transition, at a determined temperature named onset crystallization temperature $\left(T_{O}\right)$, around $85^{\circ} \mathrm{C}$, which is in good agreement with 
the DSC results. The endset temperature $\left(T_{e}\right)$ of the crystallization phenomenon is taken as the temperature reached when the increase in both viscoelastic modulus achieve a second plateau, being for the non-buried sample around $125^{\circ} \mathrm{C}$. This increase in the storage modulus indicates an increase of the rigidity of the material. The slow heating rate enhances the crystallization process during the DMTA measurement and the development of spherulites as other authors have confirmed using Thermal Optical Analysis and X-Ray Diffraction methods [30].

Significant changes due to the degradation in soil are observed by both techniques. The principal parameters evaluated for the analysis of the cold crystallization are listed in Table 2. Degradation in soil principally modifies the magnitude of the cold crystallization exotherm $\left(\Delta H_{C}\right)$, especially from 300 days of burying in soil. The increase of this parameter indicates the presence of more polymeric chains involved in the crystallization process as the degradation time is higher, thus strengthening the hypothesis of morphological changes previously drawn from the glass transition assessment.

Table 2: Calorimetric and viscoelastic parameters related to the cold crystallization.

Cold Crystallization

\begin{tabular}{cccccc}
\hline & DSC & \multicolumn{3}{c}{ DMTA } \\
\hline $\begin{array}{c}\text { Time in } \\
\text { soil (days) }\end{array}$ & $T_{0 n}\left({ }^{\circ} \mathbf{C}\right)$ & $T_{c}\left({ }^{\circ} \mathbf{C}\right)$ & $\begin{array}{c}\boldsymbol{l o g}_{E} \boldsymbol{E}^{\prime} \\
(\mathbf{P a})\end{array}$ & $T_{O}\left({ }^{\circ} \boldsymbol{C}\right)$ & $T_{e}\left({ }^{\circ} \mathbf{C}\right)$ \\
\hline $\mathbf{0}$ & $84.9 \pm 1.5$ & $123.0 \pm 1.0$ & $7.9 \pm 0.2$ & $85.5 \pm 1.0$ & $125.3 \pm 1.0$ \\
$\mathbf{3 0}$ & $84.3 \pm 1.0$ & $119.0 \pm 1.3$ & $7.8 \pm 0.3$ & $85.1 \pm 1.0$ & $125.4 \pm 1.4$ \\
$\mathbf{1 5 0}$ & $86.6 \pm 1.8$ & $125.2 \pm 1.6$ & $7.9 \pm 0.2$ & $85.0 \pm 0.8$ & $99.5 \pm 1.0$ \\
$\mathbf{3 0 0}$ & $84.3 \pm 1.6$ & $123.2 \pm 1.1$ & $7.8 \pm 0.3$ & $85.1 \pm 0.9$ & $95.6 \pm 0.6$ \\
$\mathbf{4 5 0}$ & $89.9 \pm 1.3$ & $119.7 \pm 1.2$ & $7.7 \pm 0.1$ & $85.0 \pm 0.7$ & $93.6 \pm 1.2$ \\
\hline
\end{tabular}


Changes are also observed in the mechanical spectra; the rubbery plateau is narrower as samples are more degraded, since the crystallization ends at lower temperatures. These results may suggest that the degradation in soil of the amorphous phase regions is forming shorter chains that easyly rearrange in spherulites, since the mobility is enhanced by the availability of more free volume.

\subsection{Melting characterization}

The study of the PLA melting process allows the determination of new parameters that will reinforce the knowledge of the degradation in soil effects on PLA morphology and thermal properties. The melting process of non-buried PLA has been analysed by the deconvolution of the melting endotherms that represent two different crystalline distributions. The lowest melting temperature peak, corresponding to the population with lower size (peak 1) is located at $151^{\circ} \mathrm{C}$ and the highest, corresponding to crystalline conformations with larger size (peak 2), appears at $155^{\circ} \mathrm{C},\left(T_{\mathrm{m} 1}\right)$ and $\left(T_{\mathrm{m} 2}\right)$ respectively. Figure 7 represents the evolution of both melting $\left(\Delta H_{m}\right)$ and coldcrystallization $\left(\Delta H_{c}\right)$ enthalpies along the degradation test. It is obtained that the melting enthalpy slightly differs from the immediately previous cold-crystallization enthalpy regardless the time of the experiment, confirming that PLA is initially amorphous. Higher degradation time leads to equally increase both enthalpies: as can be seen, the initially melting enthalpy was around $7 \mathrm{~J} / \mathrm{g}$ and at 300 days it reached $18 \mathrm{~J} / \mathrm{g}$ but it is up to this time when the increase is more significant, achieving at 450 days, $30 \mathrm{~J} / \mathrm{g}$. 


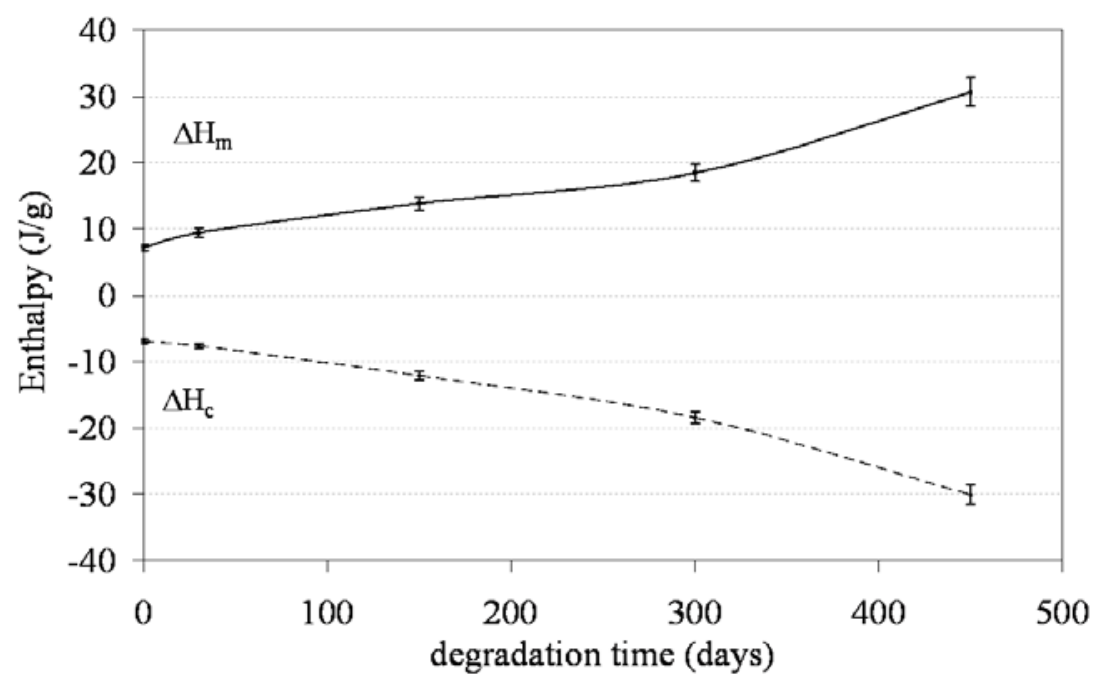

Figure 7: Crystallization and melting enthalpies vs. degradation time

Nonetheless, it has been considered interesting to perform an accurate study of the morphology of the cold crystallites obtained. Hence, from the obtained results of the melting process during the first scan, the lamellar thickness distribution " $l_{c}$ " (in Ámströngs $\left(1 \dot{A}=10^{-10} \mathrm{~m}\right)$ of the newly grown crystallites was calculated by means of Thompson equation.

$$
l_{c}=\frac{2 \cdot \sigma_{e}}{\Delta h_{m}^{\infty} \cdot\left(1-\frac{T}{T_{m}^{o}}\right)}
$$

where: $T=$ observed melting temperature $(K), T_{m}^{o}=$ equilibrium melting temperature $(K), \sigma_{e}=$ free surface energy of the basal plane $\left(\mathrm{J} \cdot \mathrm{m}^{-2}\right)$ and $\Delta h_{m}^{\infty}=$ melting enthalpy per volume unit for a crystalline phase $\left(\mathrm{J} \cdot \mathrm{m}^{-3}\right)$. For PLA the values used for calculating the lamellar thickness are, $T_{m}{ }^{0}=480 \mathrm{~K}, \sigma_{e}=60.89 \cdot 10^{-3} \mathrm{~J} \cdot \mathrm{m}^{-2}$, and $\Delta h_{m}^{\infty}=111.083 \cdot 10^{8} \mathrm{~J} \cdot \mathrm{m}^{-3}$ [31]. According to the Thompson equation, Eder assumes that at a given temperature for a sample of molten polymer, the rate of heat consumption is proportional to the fraction of lamellar which thickness is $l_{c}$ [32]. For the non-buried material, $l_{c}$ relays between 75 and $115 \AA$, the plot has been done subtracting the baseline of the endotherm 
and normalized to the maximum value of the enthalpy. The influence of degradation in soil on the lamellar thickness of the crystallites is shown at Figure 8. The plots slightly shift to higher $l_{c}$ values with longer degradation in soil, showing the continuous formation of crystalline zones with higher lamellar size. The lamellar thickness distribution is splitting into two different shoulders. A multimodal endothermic behavior, attributed to a segregation of the initial crystalline distribution into two main populations can be observed. A deconvolution procedure was applied to the melting thermograms in order to individually characterize the behavior of each population and their contribution to the overall effect using a partial areas study [33]. The following expression was employed as deconvolution.

$$
l_{c}(T)=\sum_{i} A_{i} \cdot \exp \left(\frac{-\left(T-T_{i}\right)^{2}}{\frac{w_{i}^{2}}{\ln (16)}}\right)
$$

where $T_{i}$ represents the average value of the temperatures range considered; $A_{i}$ is the maximum intensity of the curve; $w_{i}$ acts as a dispersion parameter. Deconvoluted peak temperatures, as well as the lamellar thickness at the maximum of both populations are gathered at Table 3 . As can be seen, $T_{m 1}$ remains almost constant and $T_{m 2}$ slightly increases with degradation. The areas of the individual curves have been analyzed and the relative values (listed at Table 3 ) indicate that degradation in soil promotes the growing of the lamellar distribution with lower crystalline sizes. These results indicate that as a consequence of the degradation, the heterogeneity of this material increase. As mechanical results, DSC technique has shown the crystallization process as an important indicator of the samples degradation. 


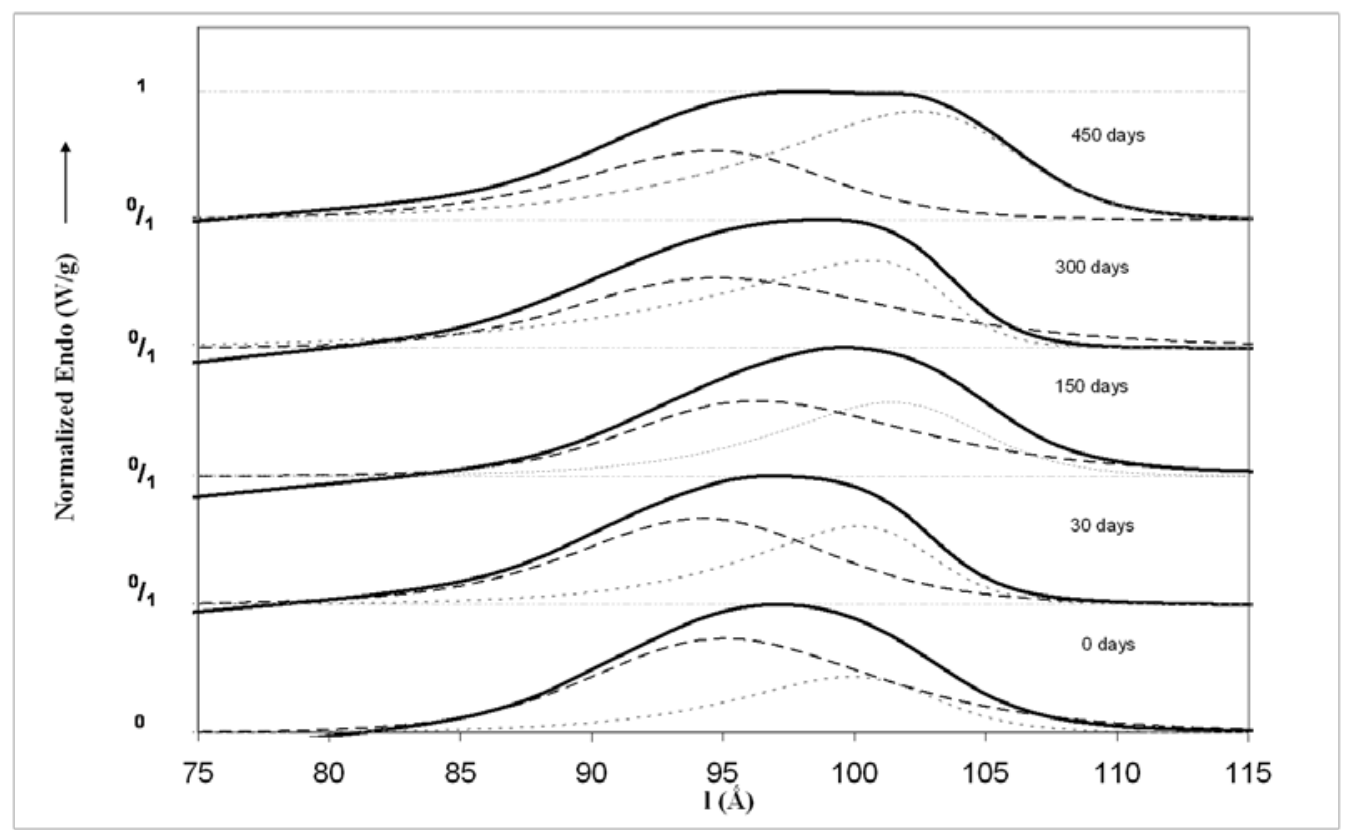

Figure 8: Influence of degradation in soil on the lamellar thickness distribution of polylactide- ( ) overall endotherm, (-) $1^{\text {st }}$ deconvoluted lamellar thickness distribution with lower crystalline sizes and (---) $2^{\text {nd }}$ deconvoluted lamellar thickness distribution with higher crystalline sizes.

Table 3: Calorimetric parameters related to the melting transition.

\begin{tabular}{ccccccc}
\hline \multicolumn{7}{c}{ Melting } \\
\hline $\begin{array}{c}\text { Time in } \\
\text { soil (days) }\end{array}$ & $T_{m 1}\left({ }^{\circ} \boldsymbol{C}\right)$ & $T_{m 2}\left({ }^{\circ} \boldsymbol{C}\right)$ & $\boldsymbol{I}_{\max 1}(\tilde{A})$ & Area $_{1}$ & $\boldsymbol{I}_{\max 2}(\tilde{A})$ & Area $_{2}$ \\
\hline $\mathbf{0}$ & $151.6 \pm 2.0$ & $154.9 \pm 2.0$ & $101.3 \pm 1.4$ & $4.5(39 \%)$ & $93.7 \pm 1.2$ & $7.0(61 \%)$ \\
$\mathbf{3 0}$ & $151.7 \pm 1.8$ & $155.1 \pm 1.4$ & $101.6 \pm 1.2$ & $6.8(44 \%)$ & $94.5 \pm 0.8$ & $8.5(56 \%)$ \\
$\mathbf{1 5 0}$ & $151.8 \pm 1.6$ & $155.6 \pm 1.7$ & $102.7 \pm 1.1$ & $8.6(51 \%)$ & $94.4 \pm 1.0$ & $8.6(49 \%)$ \\
$\mathbf{3 0 0}$ & $151.4 \pm 1.2$ & $154.9 \pm 2.0$ & $102.6 \pm 0.8$ & $10.8(55 \%)$ & $92.8 \pm 1.2$ & $8.7(46 \%)$ \\
$\mathbf{4 5 0}$ & $151.8 \pm 1.3$ & $156.9 \pm 1.3$ & $104.5 \pm 0.9$ & $11.6(63 \%)$ & $95.9 \pm 0.8$ & $6.8(37 \%)$ \\
\hline
\end{tabular}

Summing up, the increase of the relative structural relaxation enthalpy and the thermal expansion coefficient manifest the chain cleavages induced by degradation in soil of PLA. Elevate free volume of the molecular chains that form the amorphous phase allows a major mobility of the free chains. The apparition of shorter chains is 
monitored by the continuous increase in the cold-crystallization enthalpy, especially after 300 days of burying. The raise of crystallization is confirmed by the increase of the relative area of the melting endotherm related to the coldly-formed crystalline population with higher lamellar thickness.

Results provided by Thermal Analysis have been correlated with the obtained by directly measuring the molecular weight. Figure 9 shows the behaviour of average molecular weight in number and weight ( $\bar{M}_{n}$ and $\bar{M}_{w}$, respectively) as a function of the days in soil. A slight increase in the molecular weight after 30 days due to the rearrangements that PLA underwent in contact with water and soil [34] is shown. The molecular weight continuously decreases from 30 days on and it specially drops after 300 days, reaching a $50 \%$ decrease of the molecular weigh of PLA at 450 days.

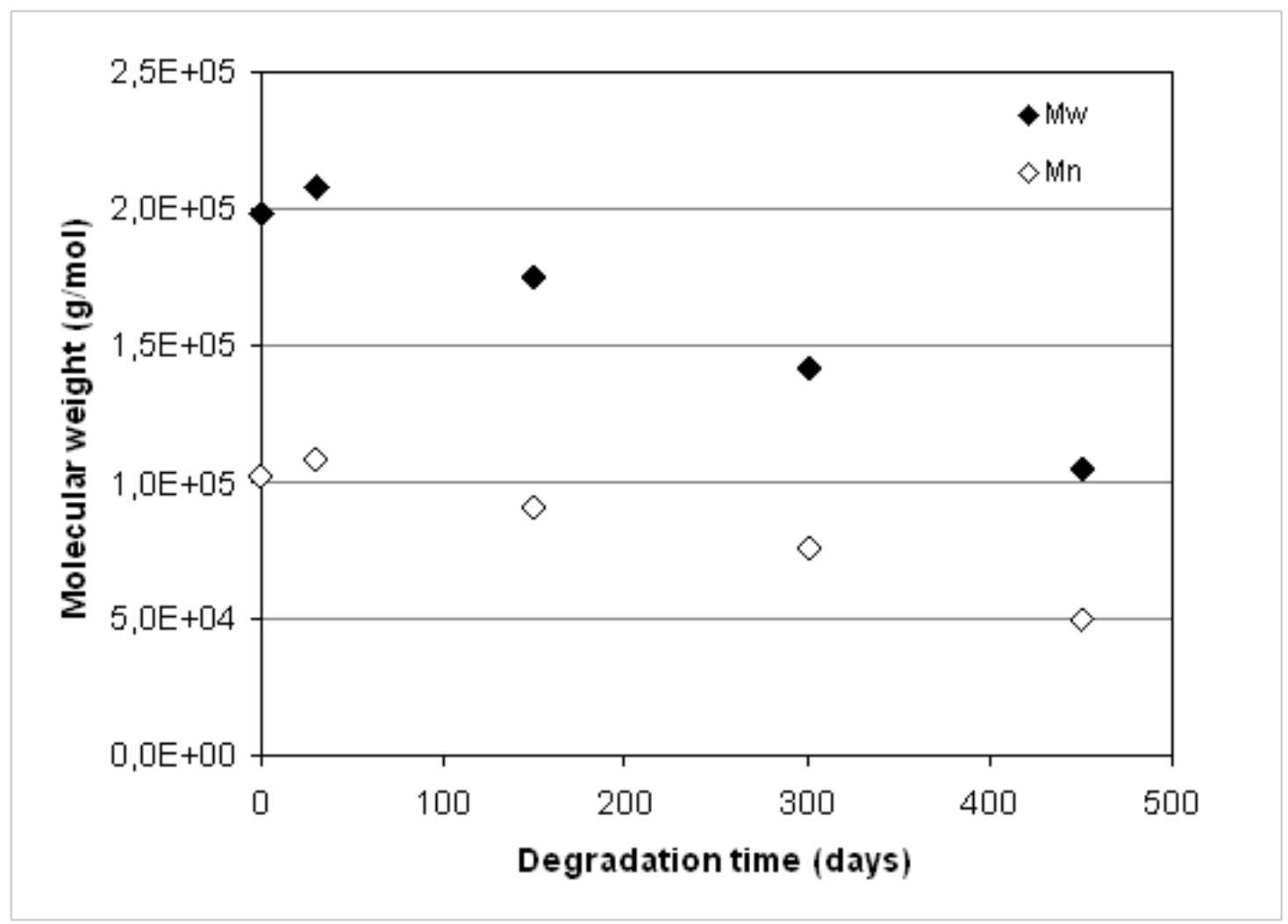

Figure 9: $\bar{M}_{n}$ and $\bar{M}_{w}$ evolution calculated by GPC. 
The correlation of Thermal Analysis assessment with the molecular weight characterization has been therefore shown to be complementary and interesting, since the stage of degradation can be effectively examined by both types of analysis. DSC and DMTA techniques are very sensitive to molecular rearrangements happening before large-scale degradation processes, and therefore capable of explaining the morphological changes induced during degradation in soil, even when no significant changes in molecular weight have occurred. Likewise, Thermal Analysis techniques have effectively monitored the effects of burial when the molecular weight experiences

a remarkable decrease, thus confirming the suitability of these techniques for understanding the influence of degradation in soil on polylactide.

\section{Conclusions}

Polylactide was buried in active soil in order to simulate its disposal stage following an international standard, and the changes in their physical properties were assessed by Differential Scanning Calorimetry and Dynamical- Mechanical Thermal Analysis.

Along the degradation in soil process, indicators such as the relative structural relaxation enthalpy and the thermal expansion coefficient have given an idea about the free volume generated within the amorphous matrix. Onset, endset and peak cold crystallization temperatures and enthalpies showed the apparition of a new crystalline phase, due to the liability of shorter chains to recombine after cleavages induced by degradation in soil. The melting temperatures and the lamelar thickness distribution shown that, especially after 300 days of burial, the crystalline fraction experiences a change from a unimodal towards a multimodal melting behaviour, coinciding with the macroscopic molecular weight decrease. These facts stress the relation between the heterogeneity acquired by PLA after chain scissions and the notable loss of properties due to a remarkable molecular weight reduction. 
The combination of both Thermal Analysis and Molecular Weight Characterization stands out as a very interesting option for characterizing not only the macroscopic changes on PLA structure induced by degradation in soil, but also for establishing new insights on the morphological rearrangements involved during the whole process.

\section{Acknowledgements}

Partial funding of this work by Ministerio de Educación y Ciencia, Project ENE200767584-C03-02 and for a pre-doctoral grant scholarship FPU to J.D. Badía, and by the Ministerio de Ciencia e Innovación for a pre-doctoral grant scholarships FPI to L. Santonja Blasco and Rosana Moriana, is gratefully acknowledged.

\section{References}

[1] Doi Y, Fukuda K, editors. Biodegradable plastic and polymers. Amsterdam (The Netherlands): Elsevier; 1994.

[2] Scott G editor, Biodegradable Polymers. Principles and applications, $2^{\text {nd }}$ ed. Dordrecht, The Netherlands: Kluwer Academic Publishers, 2002.

[3] Vert M, Schwach G, Coudane J. Present and future of PLA polymers. J Macromol Sci Pure Appl Chem 1995;A32:787-96.

[4] De Jong SJ, Arias ER, Rijkers DTS, van Nostrum CF, Kettenes-van den Bosch J J, Hennink W E. New insights into the hydrolytic degradation of poly(lactic acid): articipation of the alcohol terminus Polymer 2001;42:2795-2802.

[5] Kronenthal RL, Oser Z, Martin E, editors. Polymers in medicine and surgery. New York, NY: Plenum Press 1975;119-37. 
[6] Li S, Garreau H, Vert M. Structure-property relationships in the case of the degradation of massive aliphatic poly-( $\alpha$-hydroxy acids) in aqueous media. J Mater Sci Mater Med 1990;1:123-30.

[7] Migliaresi C, Fambri L, Cohn D. A study on the in vitro degradation of poly(lactic acid). J Biomater Sci Polym Ed 1994;4:591-606.

[8] Södergård A, Selin J-F, Näsman JH. Hydrolytic degradation of the peroxide modified poly (L-lactide). Polym Degrad Stab 1996;51:351-59.

[9] Tsuji H, Mizuno A, Ikada Y. Properties and morphology of poly(L-lactide). III. Effects of initial crystallinity on long-term in vitro hydrolysis of high molecular weight poly(L-lactide) film in phosphate-buffered solution. J Appl Polym Sci 2000;77:1452-64.

[10] Tsuji H, Ikada Y. Properties and morphology of poly(L-lactide) 4. Effects of structural parameters on long-term hydrolysis of poly(L-lactide) in phosphate-buffered solution. Polym Degrad Stab 2000;67:179-89.

[11] Hakkarainen M. Aliphatic polyesters: abiotic and biotic degradation and degradation products. Adv Polym Sci 2002;157:113-38.

[12] Shogren RL, Doane WM, Garlotta D, Lawton JW, Willett JL. Biodegradation of starch/polylactic acid/poly(hydroxyester-ether) composite bars in soi Polym Degrad Stab 2003;79:405-11. 
[13] Torres A, Li SM, Roussos S, Vert M. Poly(lactic acid) degradation in soil or under controlled conditions ACS Symp Ser 1999;7:23:218-26.

[14] Urayama H, Kanamori T, Kimura Y. Properties and biodegradability of polymer blends of poly(l-lactide)s with different optical purity of the lactate units. Macromol Mater Eng 2002;287:116-21.

[15] Siepmann J, Göpferich A. Mathematical modeling of bioerodible, polymeric drug delivery systems. Adv Drug Delivery Rev 2001;48(2-3):229-47.

[16] Schliecker G, Schmidt C, Fuchs S, Kissel T. Characterization of a homologous series of d,l-lactic acid oligomers; a mechanistic study on the degradation kinetics in vitro. Biomaterials 2003;24:3835-44.

[17] Aso Y, Yoshioka S, Li Wan Po A, Terao T. Effect of temperature on mechanisms of drug release and matrix degradation of poly(DL-lactide) microspheres. J Controlled Release 1994;31(1):33-9.

[18] Ho KLG, Pometto III AL, Hinz PN. Effects of temperature and relative humidity on polylactic acid plastic degradation. J. Environ Polym Degrad 1999;7:83-92.

[19] Calmon A, Guillaume S, Bellon-Maurel V, Feuilloley P, Silvestre F. Evaluation of material biodegradability in real conditions-development of a burial test and an analysis methodology based on numerical vision. J Environ Polym Degrad 1999;7:157-66. 
[20] H. S. Iman (1990). Adhesive properties of a symbiotic bacterium from a woodboring shipworm Appl. Environ. Microbiol. 56, 1317-22.

[21] Goheen RP, Wool RP. Degradation of polyethylene-starch blends in soil J of Environ Polym Degr 1991;42:2691-2701.

[22] Santonja-Blasco L, Contat-Rodrigo L, Moriana-Torro R, Ribes-Greus A. Thermal characterization of polyethylene blends with a biodegradable masterbatch subjected to thermo-oxidative treatment and subsequent soil burial test. J Appl Polym Sci 2007;106:2218-30.

[23] Moriana-Torró R, Contat-Rodrigo L, Santonja-Blasco L, Ribes-Greus A. Thermal characterisation of photo-oxidized HDPE/Mater-Bi and LDPE/Mater-Bi blends buried in soil. J Appl Polym Sci 2008;109:1177-1188.

[24] Contat-Rodrigo L, Ribes-Greus A, Díaz-Calleja R. Characterization by thermal analysis of PP with enhanced biodegradability. J Appl Polym Sci 2001;82:2174-84.

[25] Vallés-Lluch A, Contat-Rodrigo L, Ribes-Greus A. Influence of previous annealing on the first stage of degradation of LDPE-Mater-Bi blends aged in soil. Comparative study by thermal analysis. J Appl Polym Sci 2003;90:3359-73.

[26] Contat-Rodrigo L, Ribes-Greus A. Viscoelastic behavior of degradable polyolefins aged in soil J Appl Polym Sci 2000;78:1707-20. 
[27] ISO 846, 1997. Plastics-determination of behaviour under the action of microoganisms. Evaluation by visual examination or measurement of changes in mass or physical properties.

[28] Vogel H. The temperature dependence law of the viscosity of fluids .Phys Z 1921;22:645-46.

[29] Heya T, Okada H, Ogawa Y, Toguchi H. In-vitro and in-vivo evaluation of thyrotropin-releasing-hormone release from copoly(DL-lactic/glycolic acid) microspheres. J Pharm Sci 1994;83(5):636-40.

[30] Pluta M. Morphology and properties of polylactide modified by thermal treatment, filling with layered silicates and plasticization Polymer 2004;45:8239-51.

[31] Vasanthakumari R, Pennings AJ. Crystallization kinetics of poly(L-lactic acid). Polymer 1983;24:175-78.

[32] Wlochowicz A; Eder M. Distribution of lamella thicknesses in isothermally crystallized polypropylene and polyethylene by differential scanning calorimetry Polymer 1984;25:1268.

[33] Badía JD, Vilaplana F, Karlsson S, Ribes-Greus A.Thermal analysis as a quality tool for assessing the influence of thermo-mechanical degradation on recycled poly(ethylene terephthalate). Polymer Testing 2009;28:169-75. 
[34] Liua X, Zoub Y, Lia W, Caoa G, Chen W. Kinetics of thermo-oxidative and thermal degradation of poly(D,L-lactide) (PDLLA) at processing temperature. Polymer

Degradation and Stability 2006; 91(12):3259-65.

\section{ANNEX. OPEN-ACCESS POLICIES}

One journal found when searched for: 0141-3910

Polymer Degradation and Stability (ISSN: 0141-3910)

ROMEO: This is a RoMEO green journal

Paid OA: A paid open access option is available for this journal.

Author's Pre-print: $\checkmark$ author can archive pre-print (ie pre-refereeing)

Author's Post-print: $\checkmark$ author can archive post-print (ie final draft post-refereeing)

Publisher's Version/PDF: $\mathbf{X}$ author cannot archive publisher's version/PDF

General Conditions:

- Pre-print allowed on any website or open access repository

Voluntary deposit by author of authors post-print allowed on authors' personal website, arxiv.org or institutions open scholarly website including Institutional Repository, without embargo, where

Peposit due to Funding Body, Institutional and Governmental policy or mandate only allowed where separate agreement between repository and the publisher exists.

Set statement to accompany deposit

Published source must be acknowledged

: Must link tojournal home page or articles' DOI

Articles in some journals can be made Open Acc

Paid Open Access: Open Access Articles

Notes: . Publisher last contacted on 18/10/2013

Copyright: Article Postina Policies - Rights \& responsibilities - Eunding Body Acreements - Green Open Access - Open Access License Policy Green Open Access - Elsevier Journal Specific Embarco Periods

Updated: 16 -May-2014 - Sugoest an undate for this recore

Link to this page: hittp///nww.sherpa.ac.uk/romealissn/0141-3910 